María Jimena Berezovsky*

\title{
Sistematización de la dialéctica del paisaje para la gestión participativa del espacio público ${ }^{1}$
}

\section{Cómo citar:}

Berezovsky, M. (2020). Sistematización de la dialéctica del paisaje para la gestión participativa del espacio público. Designia, 8(1), 161-173.

\author{
${ }^{1}$ El presente artículo se deriva de la investigación desarrollada para \\ conseguir el título de Magíster en Planificación y Diseño del Paisaje \\ de la Universidad Católica de Córdoba, Argentina. \\ * Arquitecta, Magíster en Planificación y Diseño de la Universidad \\ Católica de Córdoba (UCC-MAP) - Argentina. \\ E-mail: jimebere@gmail.com \\ ORCID: https://orcid.org/0000-0002-5786-0461
}

Resumen:

\section{Palabras clave:}

Paisaje, SIG, planificación, gestión, espacio público.

\section{Key words:}

Landscape, GIS, planning, management, public Space.

\section{Recibido: 26/08/2019}

Aceptado: 30/01/2020

Para la sección Disertaciones sobre Diseño del Vol 8, № 1 de la revista Designia, la autora invitada presenta un artículo corto de reflexión, que se deriva del proyecto de investigación adelantado para conseguir el título de Magíster en Planificación y Diseño del Paisaje, de la Universidad Católica de Córdoba, Argentina.

En el texto, se reflexiona acerca de la enseñanza de la temática del paisaje en ámbitos universitarios, a partir del uso de herramientas digitales. De acuerdo con lo anterior, se presenta una propuesta pedagógica que incluye el diseño de un manual y un curso, para la planificación y adecuada intervención del paisaje. 


\begin{abstract}
:
For the section Dissertations on Design of Vol 8, No. 1 of Designia magazine, the invited author presents a short reflection article, which derives from the advanced research project to obtain the Master's Degree in Landscape Planning and Design, from the Catholic University of Córdoba, Argentina.
\end{abstract}

The paper, discuss about teaching of landscape issues in university settings, based on the use of digital tools. In accordance with the above, a pedagogical proposal includes the design of a manual and a course, for the planning and adequate intervention of the landscape.

Introducción

"El espacio es un híbrido, un mixto, donde sistema de objetos y sistema de acciones, donde fijos y flujos, donde trabajo vivo y trabajo muerto, donde forma y función, donde configuración territorial y relaciones sociales, todos ellos, son inseparables, funcionan dialécticamente y no existe uno sin el otro" (Santos, 2000, pp. 74)

El presente documento se basa en la investigación desarrollada para conseguir el título de Magíster en Planificación y Diseño del Paisaje de la Universidad Católica de Córdoba, Argentina y su posterior aplicación para la enseñanza del Paisaje en ámbitos universitarios con el uso de herramientas digitales.
En ese sentido, se elaboró un manual para la enseñanza y divulgación de la herramienta, útil para identificar y visualizar la compleja red social que se teje en cualquier región y así informarse del contexto social en el cual se va a trabajar para planificar de una mejor manera las acciones en el PAISAJE a intervenir (Imagen №1).

A este manual se le suma un curso que se va a dictar en principio 2 veces al año, para aquellas personas que quieran aprender el método.

Su uso está dirigido a Municipios, Comunas, organizaciones, centros de investigación, profesionales, académicos u otro agente con un conocimiento previo de informática, interesados en redes sociales y que estén trabajando con la gestión del paisaje. 


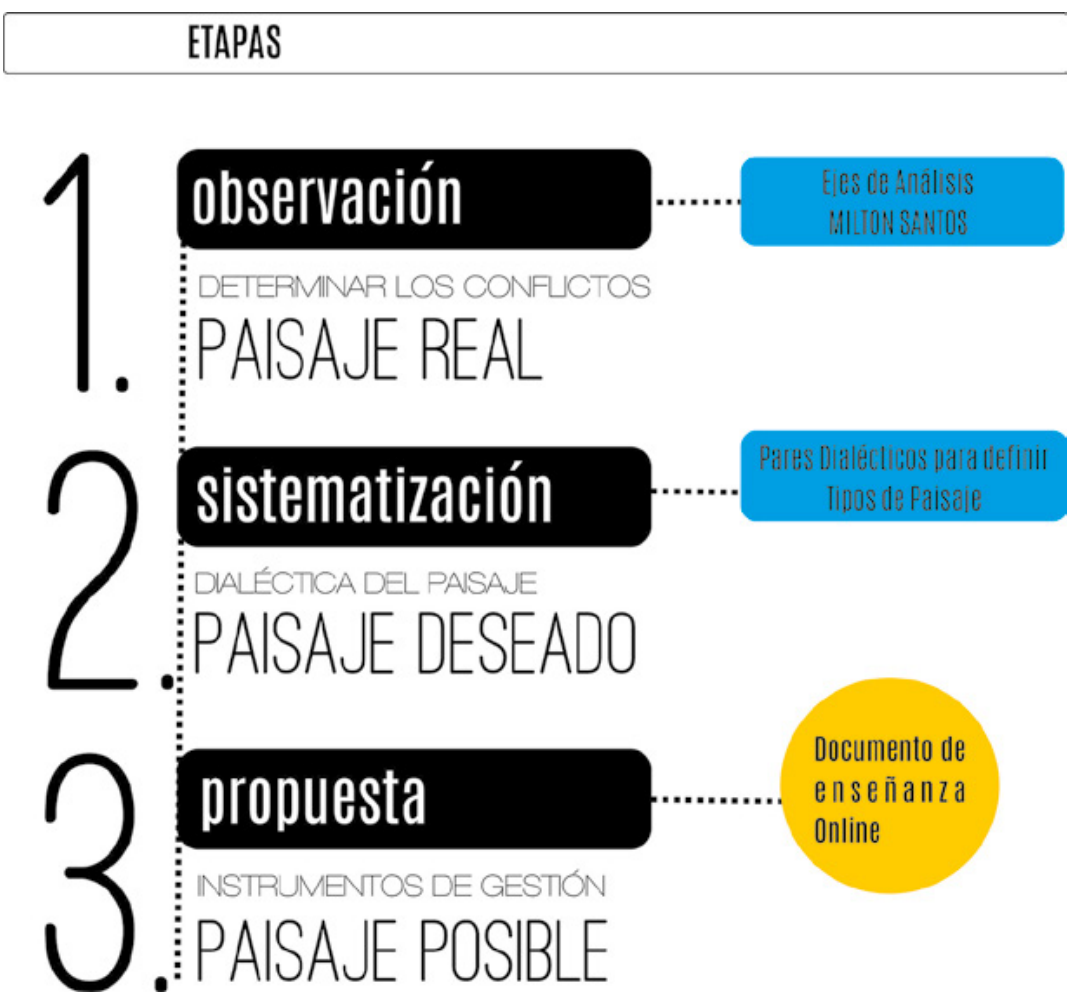

Figura 1. Ejemplos de Videos.

Fuente: autora.

Al iniciar un proyecto de planificación - en un lugar y contexto desconocido -se involucran ciertos obstáculos, siendo uno de los más críticos el desconocimiento del sistema o red social existente, es decir la estructura y características de las relaciones que forman las personas o instituciones con su entorno.

Necesariamente tanto los involucrados con la gestión de las localidades como los educadores e investigadores deben comprender este sistema para identificar con quiénes y cómo se va a trabajar, así como, comprender las relaciones que existen entre ellos.

Cuando se trabaja en estructuras sociales desconocidas existe un alto riesgo de tomar decisiones erradas, por lo que es importante invertir cierto tiempo para identificar a los actores claves y entender las relaciones sociales existentes entre ellos. Asimismo, establecer alianzas con socios que gozan de buena aceptación en el sector incrementa las posibilidades de éxito para cualquier proyecto. 
Con este documento sumado a las clases que se dieron presencialmente, se muestra paso a paso cómo realizar gráficos sociales y de valoración subjetiva que permitan dar un vistazo general de algunos escenarios y, de esta manera, facilitar la planificación de un proyecto, mediante la identificación de actores clave dentro de un entorno determinado y con la ayuda de los medios digitales.

A continuación, algunos de los métodos enseñados:
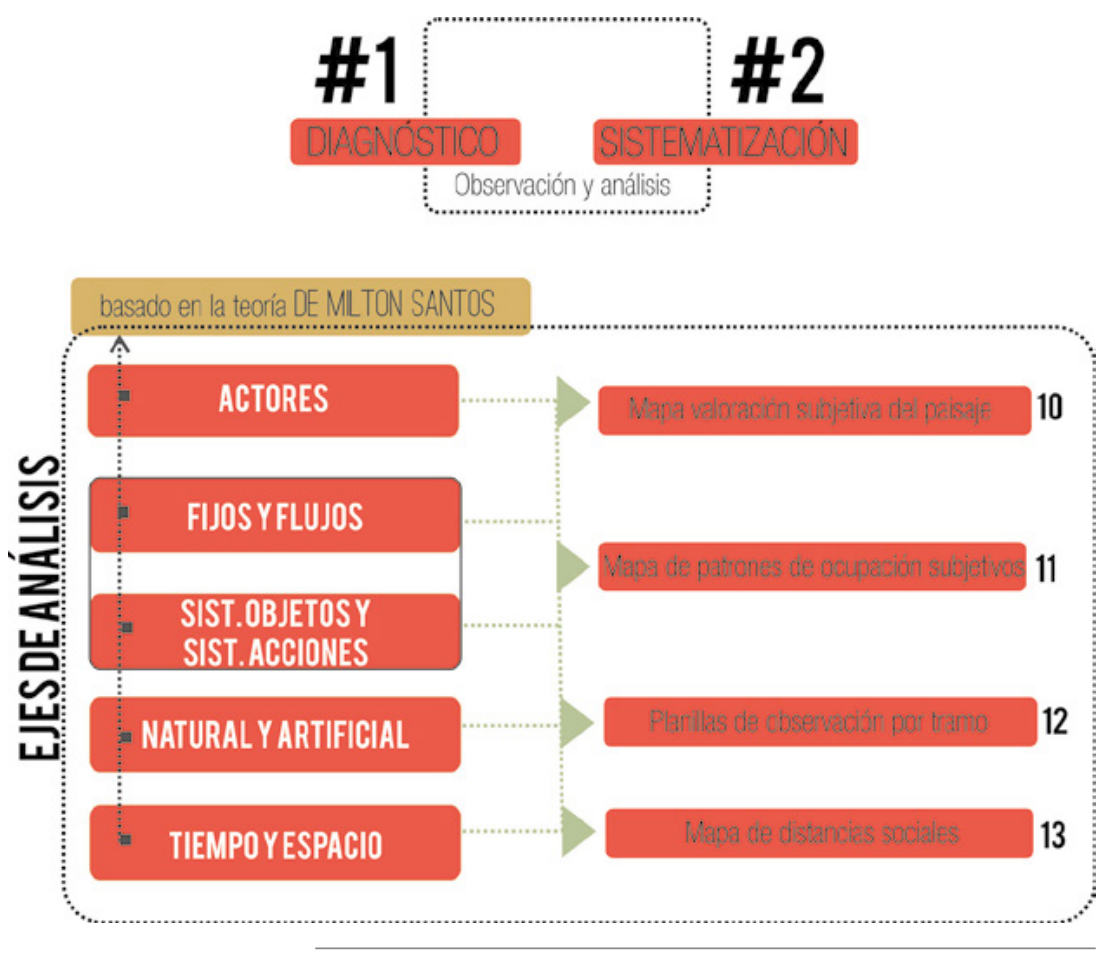

Figura 2. Ejes de análisis e instrumentos.

Fuente: autora.

Esta constituye una herramienta básica de apoyo a la toma de decisiones para la gestión de nuestros territorios, especialmente del espacio público.

Es de interés que las relaciones sociales adquieran un protagonismo especial y que sean aprehendidos por la gente, transformándose en lugares, espacios con identidad, tanto para los habitantes de la localidad como para los usuarios que la visitan y les permitan su memorabilidad, y por ello el sistema de gestión, debe estar basado en la participación activa de la comunidad local. 
${ }^{2}$ Un Sistema de Información Geográfica (SIG o GIS, en su acrónimo inglés [Geographic Information System]) es una integración organizada de hardware, software y datos geográficos diseñada para capturar, almacenar, manipular, analizar y desplegar en todas sus formas la información geográficamente referenciada con el fin de resolver problemas complejos de planificación y de gestión.
Diferentes estudios han propuesto modelos de valoración cualitativa de la calidad visual del paisaje (basados en atributos como belleza y calidad, valoración subjetiva o valor ambiental del paisaje) muy validos para resolver valoraciones concretas en determinados escenarios.

Sin embargo, aún no se ha propuesto un modelo que trate de valorar participativamente la calidad y el impacto paisajístico a través de inventarios o encuestas de preferencia de los usuarios de estos espacios.

La noción de paisaje, no solo como apariencia visual, sino como expresión de una idea, que la sustentan los componentes de estos escenarios.

El paisaje constituye una variable ambiental fundamental en la caracterización del medio ambiente, por lo que es necesario conocer y valorar su estado actual de cara a prever las posibles transformaciones que puedan tener lugar en el territorio como consecuencia de las actividades humanas.

\section{Modo de enseñanza}

Respecto al modo en que se enseñó el mapeo de estos elementos, fue mediante la aplicación de Sistemas de Información Geográfica (SIG)².

La decisión de emplear este tipo de herramientas en el estudio y enseñanza de la dialéctica de un paisaje, se debe a la capacidad de análisis del territorio físico que éstas herramientas proporcionan, así como a la posibilidad de modelizar y cuantificar diversos procesos y fenómenos, como la visibilidad desde un punto determinado o la creación de modelos predictivos. Estas capacidades hacen de los SIG una herramienta de gran utilidad en cualquier estudio relacionado con el territorio, ya sea actual, histórico o prehistórico. 
Aunque las capacidades y limitaciones son discutidas, en mi opinión, es una mera herramienta de trabajo, y no un fin de la investigación en sí misma. Evidentemente, la mejor aplicación de esta herramienta en la investigación dependerá en buena parte de su desarrollo metodológico.

Creo fundamental explicitar esta limitación puesto que, dado que los resultados arrojados por un SIG suelen tener representación cartográfica y/o cuantificable, existe una tendencia a considerarlos como válidos en sí mismos ya absolutamente exactos, cuando en realidad esos resultados estarán siempre en función de las preguntas formuladas y las variables empleadas.

\section{Mapeo de los ejes}

Ante la riqueza y variabilidad de los paisajes que se puedan observar, y tomando como base los modelos de valoración que ofrecen los SIG, surge la necesidad de proponer una nueva metodología aplicable a este tipo de territorios en la que se introduzcan componentes y características del paisaje.

Para ello se elaboraron tablas de componentes con atributos que sirven de base para elaborar cálculos y algoritmos sobre los paisajes y según lo que se quiera mostrar en ellos y de esta forma se incluya la mirada perceptual.

\begin{tabular}{|c|c|c|c|c|c|c|c|}
\hline Nombre del Actor & Tipo de Actor & Rol & $\begin{array}{c}\text { Relacion predominante } \\
\text { con el municipio }\end{array}$ & $\begin{array}{l}\text { Jerarquización de } \\
\text { su poder }\end{array}$ & Recursos & $\begin{array}{c}\text { Alcance / area de } \\
\text { cobertura }\end{array}$ & $\begin{array}{l}\text { Articulación con } \\
\text { otros actores / } \\
\text { alianzas }\end{array}$ \\
\hline Municipalidad de VSL. & Púbico & $\begin{array}{l}\text { Preveer y garantizar la prestación } \\
\text { de los servicios Cobrar tazas } \\
\text { correspondientes, } \\
\text { Responsabilizarse por la gestión }\end{array}$ & I & ALTO & $\begin{array}{l}\text { Leyes y } \\
\text { Ordenanzas }\end{array}$ & m2 & con TODOS \\
\hline Parques Temáticos & Privado & Prestar senvicios de recreación & A favor & ALTO & \begin{tabular}{|c|} 
Incorporación \\
diaria de turistas a \\
la localidad
\end{tabular} & & $\begin{array}{l}\text { con turistas y } \\
\text { ciudadanos } \\
\text { permanentes }\end{array}$ \\
\hline Turistas & Ciudadania & $\begin{array}{l}\text { Observar, conocer y apreciar la } \\
\text { historia y cultura del lugar }\end{array}$ & Indiferente & ALTO & & & $\begin{array}{c}\text { parques temáticos, } \\
\text { comercios y } \\
\text { empresa privada }\end{array}$ \\
\hline \begin{tabular}{|l} 
Residentes \\
permanentes
\end{tabular} & Ciudadania & $\begin{array}{l}\text { Vivir en un lugar donde poder } \\
\text { educarse y recrearse }\end{array}$ & A favor y en contra & MEDIO & & & $\begin{array}{c}\text { los comercios, } \\
\text { municipalidad, } \\
\text { instituciones } \\
\text { públicas y privadas }\end{array}$ \\
\hline Comerciantes & Privado & $\begin{array}{l}\text { obtener beneficios económicos } 0 \\
\text { publicitarios }\end{array}$ & A favor y en Contra & MEDIO & & & $\begin{array}{c}\text { municipalidad, } \\
\text { turistas y ciudadanos } \\
\text { permanentes }\end{array}$ \\
\hline Instituciones & Público & $\begin{array}{l}\text { Establecer lineamientos, aprobar } \\
\text { licencias, aprobar y dictar } \\
\text { ordenanzas }\end{array}$ & A Favor & ALTO & & & municipalidad \\
\hline Instituciones & Privado & $\begin{array}{l}\text { Adiministrar la concesión de los } \\
\text { servicios que presta }\end{array}$ & Indiferente & MEDIO & & & $\begin{array}{c}\text { ciudadanos } \\
\text { permanentes, } \\
\text { municialidad }\end{array}$ \\
\hline
\end{tabular}

Figura 3. Ejemplo de tabla de componentes mapeados con sus atributos. 
Para mapear en $\mathrm{Qgis}^{3}$ estos tipos de paisajes, se elaboraron grillas de atributos como la anterior (Figura №3).

Habrá tantos tipos de paisajes resultantes, como sistemas de acciones (ó usos del suelo) se les den a los objetos mapeados, y estos a su vez dependerán de quienes realicen las encuestas y de quienes las procesen.

Se le agrega a cada elemento mapeado normalmente en un mapa territorial, un atributo ${ }^{4}$ de esta tabla, tanto como en sistema de objetos como en sistema de acciones. Esta es una herramienta muy importante, por ejemplo, a la hora de elaborar mapas de uso del suelo para poder tomar decisiones de planificación en nuestros municipios ó comunas ya que no solo depende de las decisiones de los técnicos, sino que hay un fuerte compromiso de la ciudadanía participante.

Uno de los ejemplos que surgen de la aplicación de este método es el MAPA DE PATRONES DE OCUPACIÓN SUBJETIVO. Este refleja el estudio de los patrones de ocupación y uso de espacios de un conjunto de personas, o más concretamente, de las preferencias en la selección de los lugares de ocupación por parte de ese grupo, en un momento determinado, y las necesidades a las que dicha selección responde. 
Paso 1: se elabora una grilla de base (Imagen №4), donde lo más interesante de esta encuesta, es que los propios ciudadanos o usuarios de estos espacios son quienes delimitan sus propias áreas mediante el dibujo sobre un mapa.

Paso 2: se plantea una iconografía determinada para armar los relatos, de manera que una vez conformados los mapeos, estos permitan informar, señalizar, y elaborar lecturas sobre diferentes problemáticas, así como visibilizar formas articuladas de organización y transformación territorial.

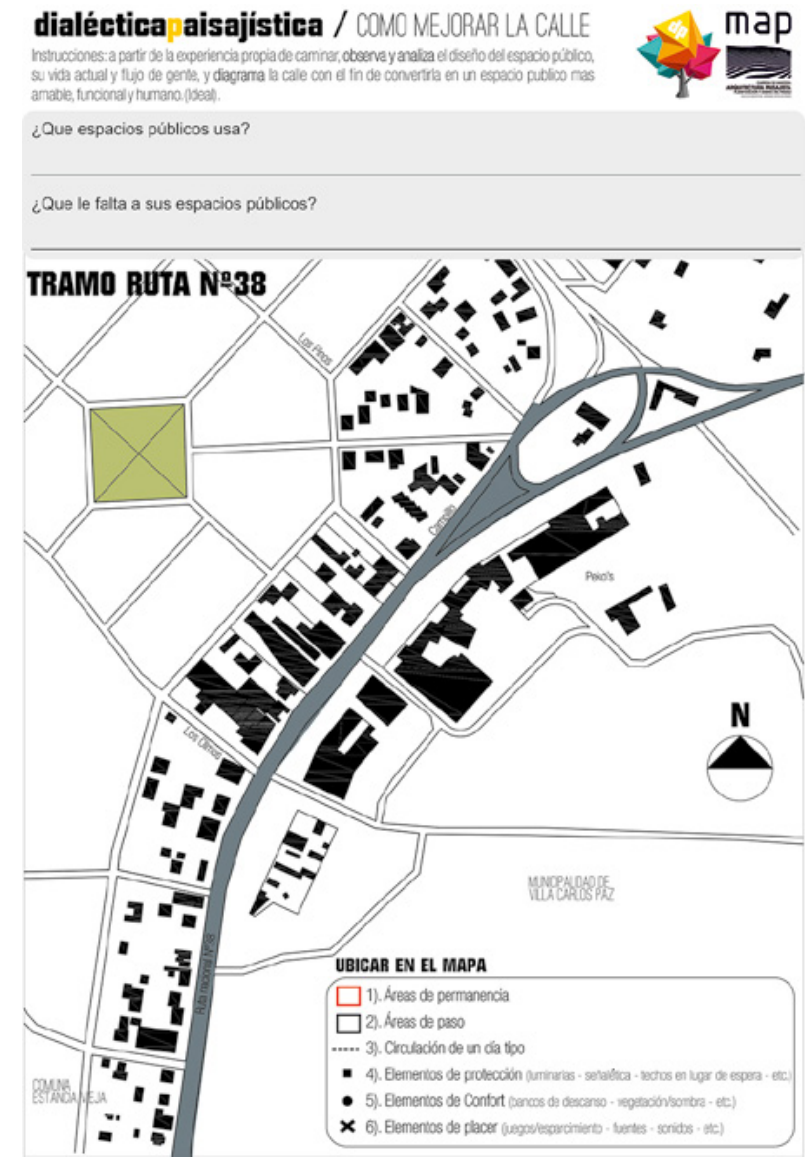

Figura 4. Ejemplo de Grilla Base para realizar la encuesta.
Paso 3: además del mapeo, se encabeza la actividad con dos preguntas que cada encuestado debe responder según sus usos y costumbres, estas respuestas permiten que los participantes rememoren zonas, espacios, rutas, lugares, itinerarios y todo aquello que consideren espacio público. Esto muestra de que manera cada persona define y caracteriza el territorio que luego mapeó individualmente.

Paso 4: la Representación. Se les entrega a los participantes, fibras ó lápices de distintos colores en función de las referencias que se proponen en la grilla, y para dar mayor libertad de expresión a quienes las realizan. 

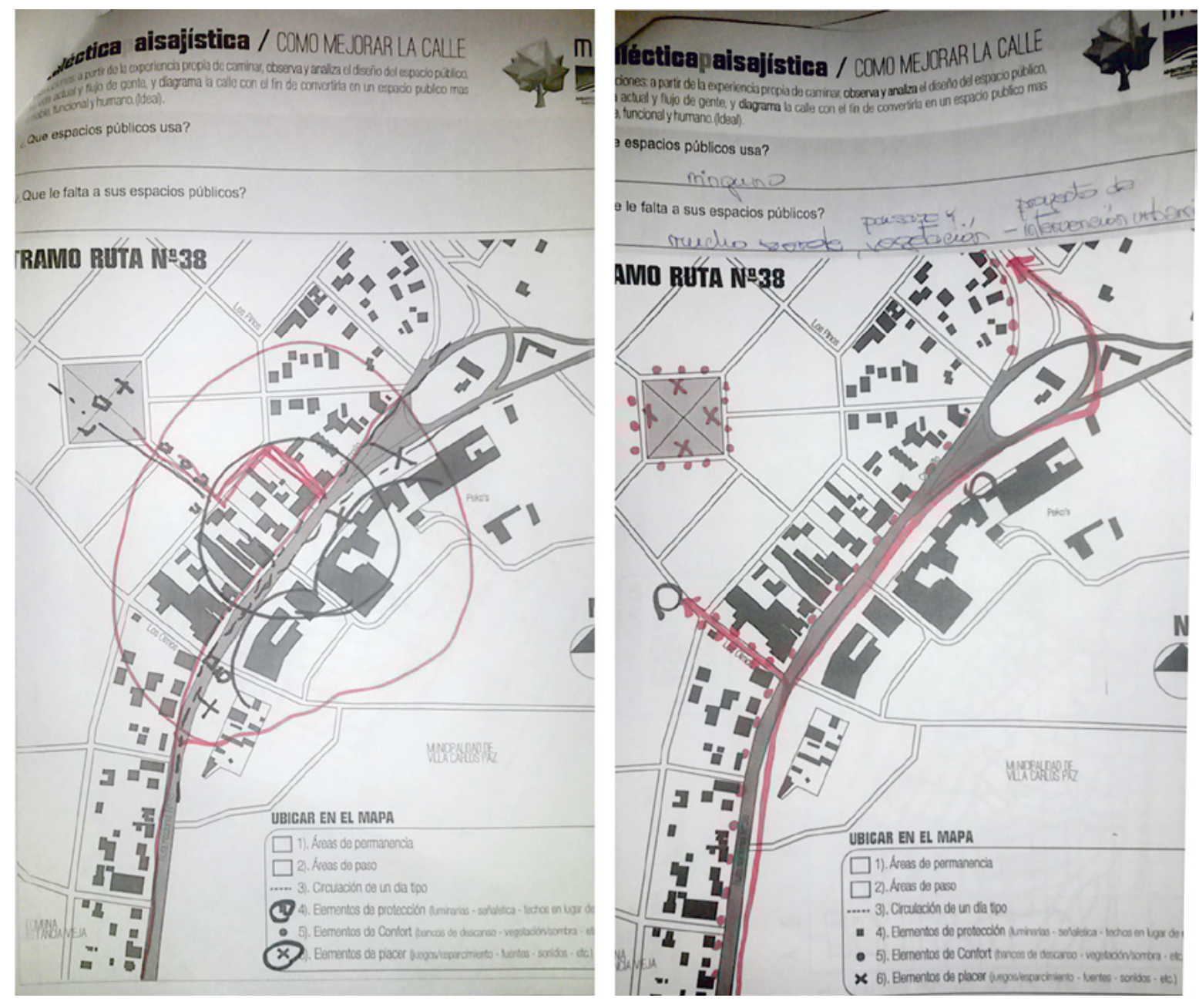

品

169

Figura 5. Ejemplo de encuestas realizadas por la comunidad.

Fuente: autora. 
Paso 5: se debe procesar cada encuesta realizada tomando criterios comunes, y respetando en mayor medida los trazos originales (Figura №6).

\section{MAPADEACTORESDEVILLASANTACRUZDELLAGO

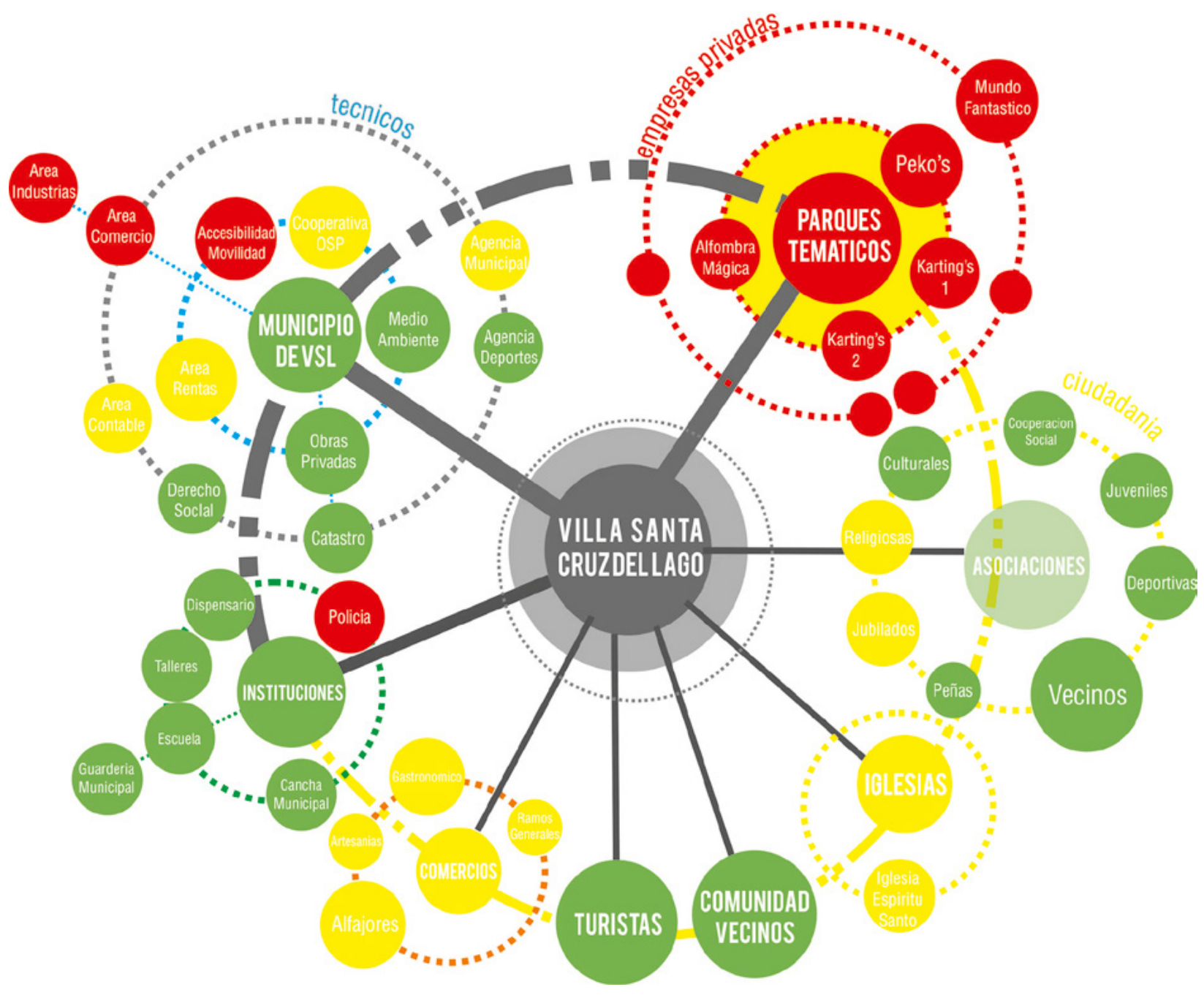



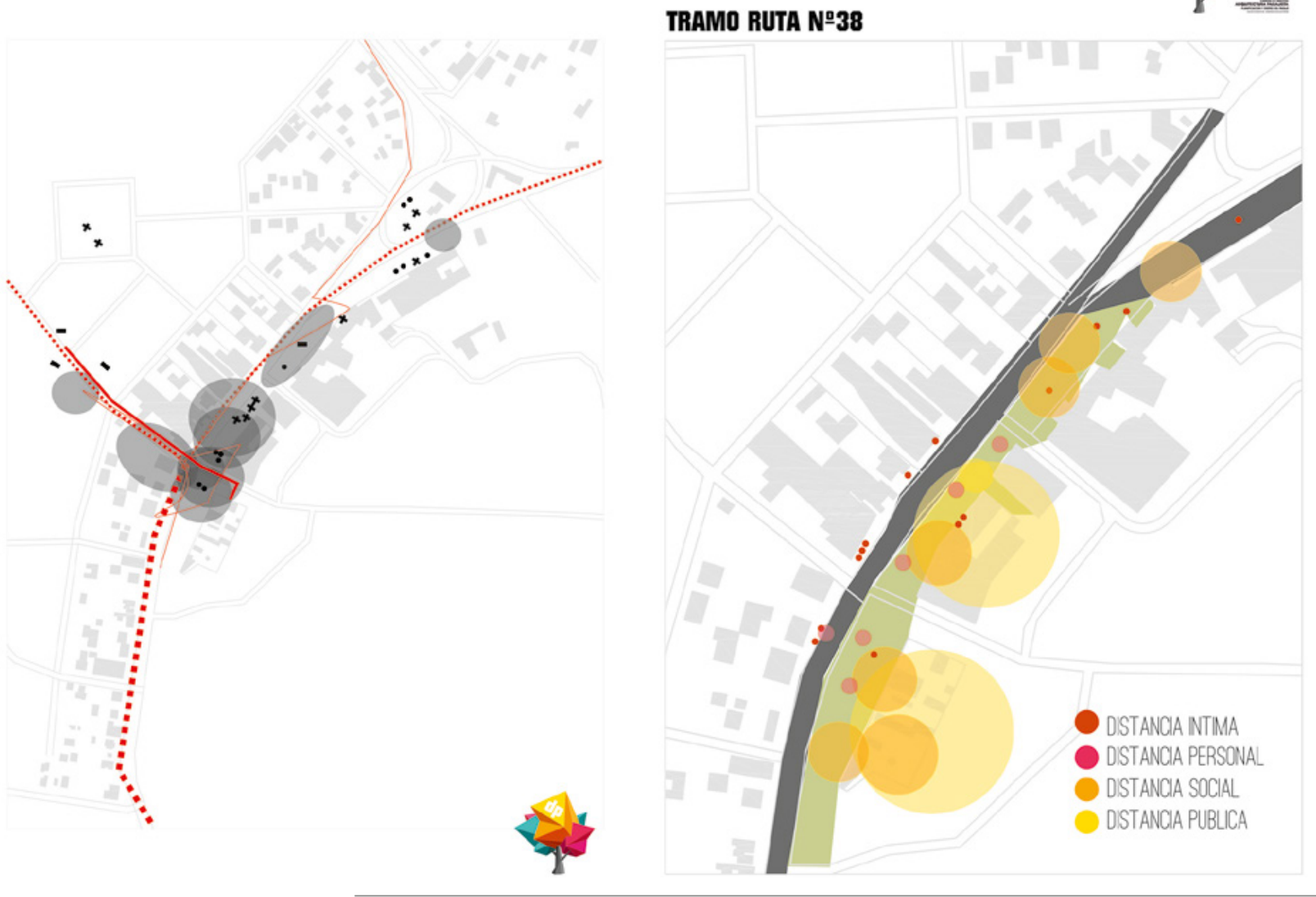

Figura 6. Ejemplo de gráficos Procesados Digitalmente. 
Se propone su digitalización y posterior vectorización con algún programa de diseño, para que no surjan errores importantes. Una vez obtenidos todos los resultados en formato digital, se procede a superponerlos con el uso de la herramienta SIG para encontrar áreas homogéneas de uso o transición dentro de los Espacios Públicos de estudio (Figura 7).
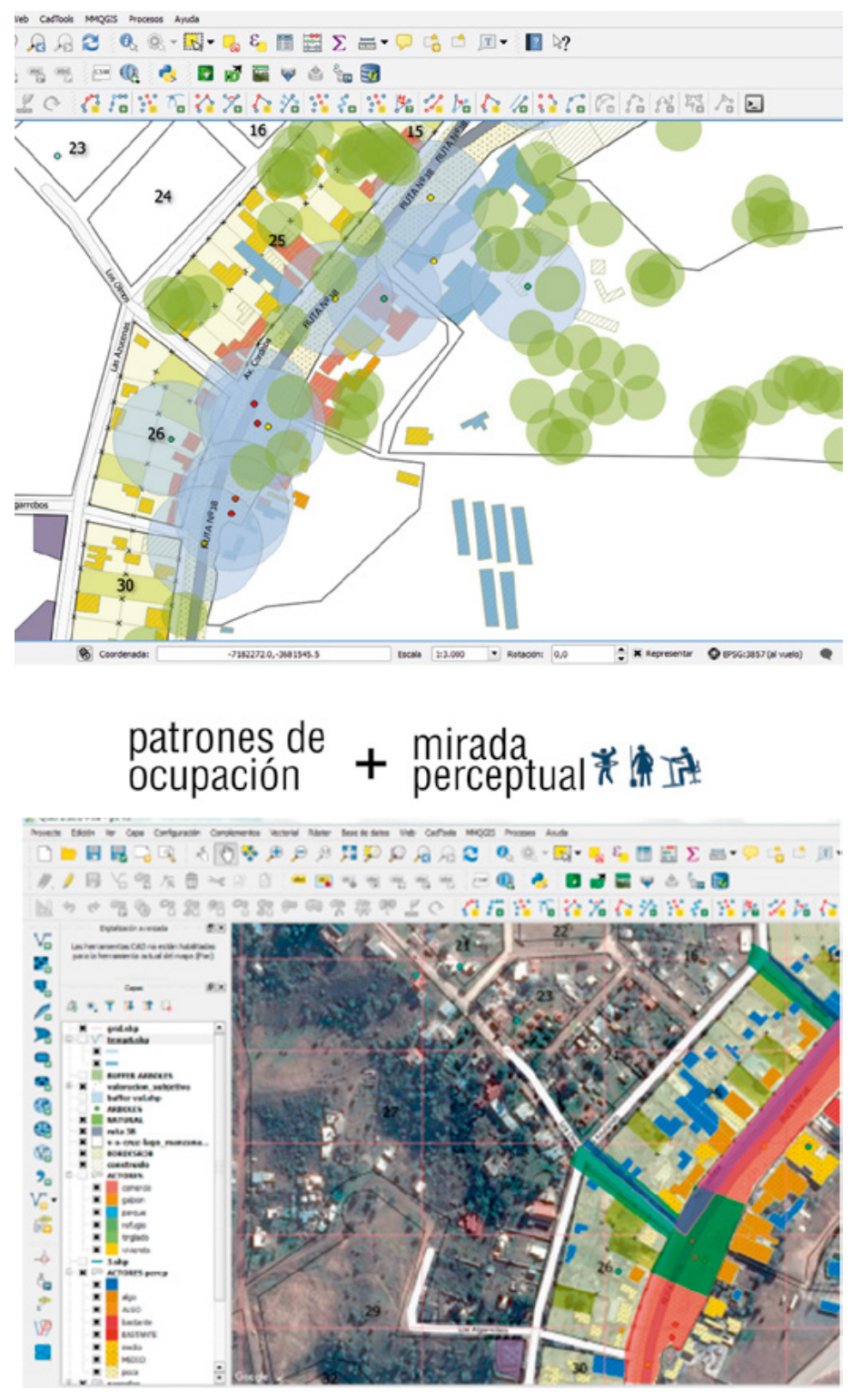

Figura 7. Digitalización de las encuestas en un Sistema de Información Geográfica. 


\section{Conclusiones}

Con esta herramienta, la idea es co-crear junto a la comunidad y el gobierno, espacios de intercambio entre todos los actores involucrados, y así poder identificar problemas, co-crear soluciones, prototiparlas, testearlas e implementarlas.

Todas las cabezas piensan mejor que una. Con este mapeo se visibilizan problemas, e invita a toda la sociedad a co-crear soluciones para resolverlos.

A partir de aquí, se pueden brindar talleres en donde se generen diagnósticos, se proyectamos soluciones y metodologías de trabajo para incentivar a más personas a convertirse en agentes de cambio.

Se fomenta la transferencia de conocimiento y espíritu de cambio en todos los sectores de la sociedad.

Esto se logra a través de eventos, festivales, talleres y charlas que se pueden realizar en los Espacios Públicos donde se pretende trabajar.

Lo más importante de este método, es culminar con un documento de gestión participativa para municipios o docentes de la temática para que puedan aplicar esta herramienta en sus ámbitos de trabajo y en contextos digitales.

\section{REFERENCIAS BIBLIOGRÁFICAS}

Santos, M. (1996). La naturaleza del espacio. Técnica y tiempo. Razón y emoción. Barcelona: Ariel. 